\title{
1,2,6-Trimethyl-3,5-nonamethylenepyridinium perchlorate. Crystal structure and molecular mechanics calculations for ansa-[9]-meta-cyclophanes
}

\author{
Teodor Silviu Balaban,*, Alexandru T. Balaban, ${ }^{\#, b}$ Sabine Foroc ${ }^{c}$, and Hans-Jörg Lindner \\ ${ }^{\mathrm{a}}$ Forschungszentrum Karlsruhe, Institut für Nanotechnologie, \\ Postfach 3640, D-76021 Karlsruhe, Germany \\ ${ }^{b}$ Texas A\&M University, Department of Marine Sciences, \\ Galveston TX 77553-1675, USA \\ ${ }^{c}$ Institut für Organische Chemie, Technische Hochschule Darmstadt, \\ Petersenstr. 22, D-64287 Darmstadt, Germany \\ E-mail: silviu.balaban@int.fzk.de; balabana@tamug.tamu.edu
}

\begin{abstract}
The nonamethylene bridge of the title compound shows high conformational mobility and an asymmetric structure in the crystal. Molecular mechanics ( $\mathrm{MM}+$ ) calculations reproduced well the crystal structure, which has the lowest energy conformation.
\end{abstract}

Keywords: 1,2,6-Trimethyl-3,5-nonamethylenepyridinium, crystal structure, ansa-[9]-metacyclophanes

\section{Introduction}

The crystal molecular structure of medium and large cyclophanic rings is an interesting subject which complements the information gained on the structure in solution by dynamic NMR methods. The low temperature NMR study of heterocyclic [9]cyclophanes has revealed a rapid ring flipping with an energy barrier $\Delta \mathrm{G} \ddagger$ between 8.9 and $9.8 \mathrm{kcal} / \mathrm{mol}{ }^{1}{ }^{1}$ In order to gain insight on the crystal structure of the nonamethylene bridge we analysed the structure of the title compound (3) by X-ray crystallography. Furthermore, we performed molecular mechanics calculations using the $\mathrm{MM}+$ force field, which is adequately parameterised for cycloalkanes within the HyperChem ${ }^{\circledR}$ package, ${ }^{2}$ in order to obtain more information on such [9]metacyclophanes annulated to related heterocycles. The calculations show that several conformations of the ansa bridge are within $1.5 \mathrm{kcal} / \mathrm{mol}$ of the lowest energy structure which corresponds to the one encountered in the crystal. 


\section{Results and Discussion}

Compounds 1-3 were previously synthesized and analysed by dynamic ${ }^{1} \mathrm{H}-\mathrm{NMR}$ techniques in the temperature range between $+20^{\circ} \mathrm{C}$ and $-90^{\circ} \mathrm{C}$ in $\mathrm{CD}_{2} \mathrm{Cl}_{2} .{ }^{1}$ The various conformations involved in the bridge flipping revealed by the NMR spectra were presented and discussed. The three compounds were the pyrylium perchlorate $\left(1, \mathrm{X}=\mathrm{O}^{+}\right)$, obtained by diacetylation of cyclododecene by acetic anhydride and $70 \%$ perchloric acid, ${ }^{3}$ the corresponding pyridine $(2, \mathrm{X}=$ $\mathrm{N})$ and $\mathrm{N}$-methylpyridinium perchlorate $\left(3, \mathrm{X}=\mathrm{N}-\mathrm{Me}^{+}\right)$formed from the pyrylium salt 1 and ammonia and methylamine, respectively. The last compound is the topic of the present paper.
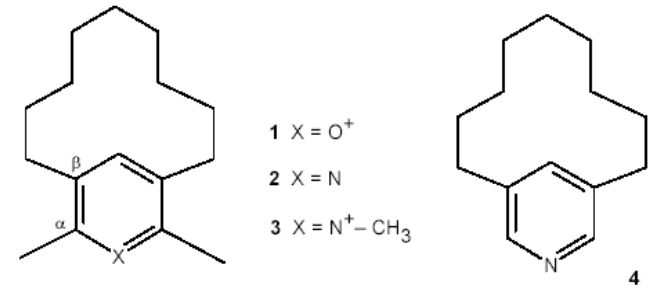

Figure 1 presents an ORTEP plot of the molecular structure of $\mathbf{3}$ in the crystal. The perchlorate anion is disordered in the crystal, and multiple orientations had to be taken into account for solving the structure. Only one of the orientations is shown in Figure 1. Figure 2 presents a different orientation of the molecule together with the unit cell and the different orientations of the distorted perchlorate anion.

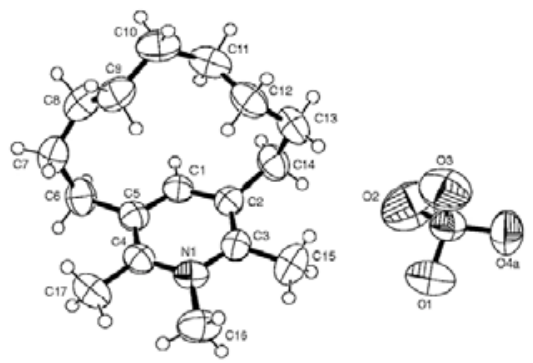

Figure 1. ORTEP plot of 3 in the crystal with atom numbering. Only the most populated orientation of the distorted perchlorate anion is shown. Note the relatively large thermal ellipsoids of the methylenic carbons in the ansa-bridge, of the three-methyl carbons and of the oxygen atoms in comparison to the relatively rigid pyridinium ring atoms.

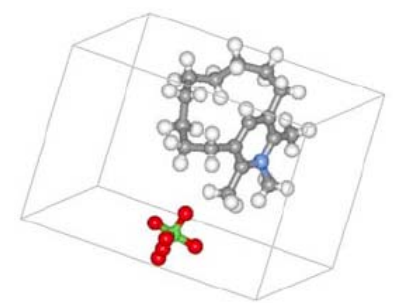

Figure 2. A different orientation of 3 for visualizing the conformation of the ansanonamethylene bridge. 
Table 1. Atomic coordinates for non-hydrogen atoms with standard e.s.d.'s in parentheses and the equivalent isotropic displacement coefficients $\left(\AA^{2}\right)$

\begin{tabular}{ccccc}
\hline Atom & $\mathrm{x}$ & $\mathrm{y}$ & $\mathrm{z}$ & $\mathrm{Ueq}$ \\
\hline $\mathrm{C} 1$ & $0.7446(4)$ & $0.6242(2)$ & $0.5568(2)$ & $0.0537(8)$ \\
$\mathrm{C} 2$ & $0.6029(4)$ & $0.6032(2)$ & $0.5331(2)$ & $0.0541(8)$ \\
$\mathrm{C} 3$ & $0.4927(4)$ & $0.6392(3)$ & $0.5864(2)$ & $0.0586(8)$ \\
$\mathrm{N} 1$ & $0.5280(3)$ & $0.6872(2)$ & $0.6632(2)$ & $0.0570(7)$ \\
$\mathrm{C} 4$ & $0.6678(4)$ & $0.6999(3)$ & $0.6912(2)$ & $0.0568(8)$ \\
$\mathrm{C} 5$ & $0.7802(3)$ & $0.6704(2)$ & $0.6361(2)$ & $0.0564(8)$ \\
$\mathrm{C} 6$ & $0.9384(4)$ & $0.6804(3)$ & $0.6620(3)$ & $0.0755(10)$ \\
$\mathrm{C} 7$ & $0.9946(4)$ & $0.5874(4)$ & $0.7153(3)$ & $0.0804(11)$ \\
$\mathrm{C} 8$ & $0.9968(4)$ & $0.4846(4)$ & $0.6650(3)$ & $0.0853(12)$ \\
$\mathrm{C} 9$ & $0.8719(5)$ & $0.4125(3)$ & $0.6843(3)$ & $0.0764(11)$ \\
$\mathrm{C} 10$ & $0.8542(5)$ & $0.3230(3)$ & $0.6176(3)$ & $0.0822(12)$ \\
$\mathrm{C} 11$ & $0.7718(5)$ & $0.3524(3)$ & $0.5358(3)$ & $0.0794(11)$ \\
$\mathrm{C} 12$ & $0.6096(5)$ & $0.3710(3)$ & $0.5506(2)$ & $0.0734(11)$ \\
$\mathrm{C} 13$ & $0.5283(5)$ & $0.4241(3)$ & $0.4741(3)$ & $0.0820(12)$ \\
$\mathrm{C} 14$ & $0.5752(5)$ & $0.5354(3)$ & $0.4526(2)$ & $0.0707(10)$ \\
$\mathrm{C} 15$ & $0.3348(4)$ & $0.6256(4)$ & $0.5632(3)$ & $0.0863(13)$ \\
$\mathrm{C} 16$ & $0.4083(5)$ & $0.7289(3)$ & $0.7183(3)$ & $0.0836(12)$ \\
$\mathrm{C} 17$ & $0.6922(5)$ & $0.7440(3)$ & $0.7803(3)$ & $0.0829(12)$ \\
C11 & $0.08209(10)$ & $0.51088(7)$ & $0.36700(6)$ & $0.0725(3)$ \\
O1 & $0.0407(4)$ & $0.5937(3)$ & $0.4224(2)$ & $0.1120(12)$ \\
O2 & $0.2245(5)$ & $0.5389(4)$ & $0.3412(3)$ & $0.156(2)$ \\
O3 & $0.0949(6)$ & $0.4166(2)$ & $0.4126(3)$ & $0.1257(13)$ \\
O4A & $-0.0519(13)$ & $0.4960(14)$ & $0.3151(12)$ & $0.147(5)$ \\
O4B & $0.0749(32)$ & $0.5136(21)$ & $0.2709(14)$ & $0.118(9)$ \\
O4C & $0.0104(13)$ & $0.5074(14)$ & $0.2930(10)$ & $0.058(4)$ \\
\hline
\end{tabular}

The final atomic coordinates for non-hydrogen atoms are given in Table 1. Selected bond lengths and angles are given in Table 2. There are no intermolecular distances shorter than van der Waals contacts. Interestingly, the structure is asymmetric as evidenced by the torsion angles of the nonamethylene bridge, which are listed in Table 3.

We could reproduce the structure by performing energy minimizations with $\mathrm{MM}+$ molecular mechanics within the HyperChem ${ }^{\circledR}$ package. The force field is adequately parameterized and the lowest energy conformation was indeed the one encountered in the crystal. However, by a combination of molecular dynamics and repeated energy minimization, several other conformations could be found. These were situated within less than $1.5 \mathrm{kcal} / \mathrm{mol}$ higher in energy. Table 3 presents the relative energies and the torsion angles of the Nonamethylene Bridge for the corresponding pyridine devoid of the 2,6- ( $\alpha$-methyl groups 4 Also given in Table 
3 are the torsion angles of the nonamethylene bridge encountered in the crystal of 3 . The next lowest energy conformation $\mathbf{4 b}$, which is also asymmetric, is only $0.5 \mathrm{kcal} / \mathrm{mol}$ higher in energy than the one, which mimics the crystal structure 4a. The next two conformations have a $\mathrm{C}_{2}$ symmetry axis passing through $\mathrm{C} 1$ and $\mathrm{C} 10$. These four structures are presented in Figure 3. Other conformations of the nonamethylenic chain, all higher in energy, were also encountered as stable minima.

Table 2. Selected bond lengths $(\AA)$ and angles $\left(^{\circ}\right)$

\begin{tabular}{llllll}
\hline C1-C2 & $1.375(5)$ & C2 C1 C5 & $122.4(3)$ & O4C C11 O3 & $116.4(8)$ \\
C1-C5 & $1.385(4)$ & C3 C2 C1 & $118.2(3)$ & O4C C11 O2 & $103.5(7)$ \\
C2-C3 & $1.376(5)$ & C3 C2 C14 & $123.0(3)$ & O3 C11 O2 & $106.3(3)$ \\
C2-C14 & $1.524(5)$ & C1 C2 C14 & $118.7(3)$ & O4C C11 O1 & $114.2(8)$ \\
C3-N1 & $1.361(4)$ & N1 C3 C2 & $119.0(3)$ & O3 C11 O1 & $111.7(2)$ \\
C3-C15 & $1.500(5)$ & N1 C3 C15 & $119.0(3)$ & O2 C11 O1 & $103.1(3)$ \\
N1-C4 & $1.361(4)$ & C2 C3 C15 & $122.0(3)$ & O4C C11 O4B & $27.8(9)$ \\
N1-C16 & $1.482(4)$ & C4 N1 C3 & $123.2(3)$ & O3 C11 O4B & $121.5(11)$ \\
C4-C5 & $1.381(5)$ & C4 N1 C16 & $118.4(3)$ & O2 C11 O4B & $75.9(11)$ \\
C4-C17 & $1.489(5)$ & C3 N1 C16 & $118.4(3)$ & O1 C11 O4B & $124.8(11)$ \\
C5-C6 & $1.509(5)$ & N1 C4 C5 & $118.6(3)$ & O4C C11 O4A & $27.4(8)$ \\
C6-C7 & $1.528(6)$ & N1 C4 C17 & $118.2(3)$ & O3 C11 O4A & $103.1(7)$ \\
C7-C8 & $1.519(6)$ & C5 C4 C17 & $123.2(3)$ & O2 C11 O4A & $130.9(7)$ \\
C8-C9 & $1.498(6)$ & C4 C5 C1 & $118.2(3)$ & O1 C11 O4A & $101.3(7)$ \\
C9-C10 & $1.538(6)$ & C4 C5 C6 & $122.3(3)$ & O4B C11 O4A & $55.2(10)$ \\
C10-C11 & $1.507(6)$ & C1 C5 C6 & $119.4(3)$ & C11 O2 O4B & $53.5(6)$ \\
C11-C12 & $1.522(7)$ & C5 C6 C7 & $113.5(3)$ & O4C O4A O4B & $2.9(23)$ \\
C12-C13 & $1.543(6)$ & C8 C7 C6 & $113.9(3)$ & O4C O4A C11 & $62.5(17)$ \\
C13-C14 & $1.520(5)$ & C9 C8 C7 & $114.9(4)$ & O4B O4A C11 & $62.2(12)$ \\
C11-O1 & $1.406(3)$ & C8 C9 C10 & $114.0(4)$ & O4C O4B O4A & $2.9(22)$ \\
C11-O2 & $1.409(5)$ & C11 C10 C9 & $114.6(3)$ & O4C O4B C11 & $63.0(18)$ \\
C11-O3 & $1.395(3)$ & C10 C11 C12 & $113.9(3)$ & O4A O4B C11 & $62.7(12)$ \\
C11-O4B & $1.470(2)$ & C11 C12 C13 & $115.3(3)$ & O4C O4B O2 & $113.1(25)$ \\
C11-O4A & $1.474(13)$ & C14 C13 C12 & $115.9(3)$ & O4A O4B O2 & $113.1(18)$ \\
C11-O4C & $1.308(12)$ & C2 C14 C13 & $113.8(3)$ & C11 O4B O2 & $50.5(9)$ \\
& & & & O4A O4C O4B & $174.2(45)$ \\
& & & & O4B O4C C11 & $89.2(23)$ \\
\hline
\end{tabular}


Table 3. MM+ Energies and torsion angles $\left(^{\circ}\right)$ of the bridge in the crystal structure of 3 with e.s.d.'s in parentheses and in the calculated pyridinophanes 4

\begin{tabular}{|c|c|c|c|c|c|c|c|c|c|c|c|}
\hline \multicolumn{2}{|c|}{$\begin{array}{l}\text { MM+ Energy } \\
(\mathrm{kcal} / \mathrm{mol})\end{array}$} & \multicolumn{2}{|c|}{$\begin{array}{c}\mathrm{C} 1 \mathrm{C} 2 \mathrm{C} 14 \mathrm{C} 13 \\
\mathrm{C} 2 \mathrm{C} 14 \mathrm{C} 13 \mathrm{C} 12\end{array}$} & \multicolumn{2}{|c|}{$\begin{array}{l}\mathrm{C} 14 \mathrm{C} 13 \mathrm{C} 12 \mathrm{C} 11 \\
\mathrm{C} 13 \mathrm{C} 12 \mathrm{C} 11 \mathrm{C} 10\end{array}$} & \multicolumn{2}{|c|}{$\begin{array}{c}\mathrm{C} 12 \mathrm{C} 11 \mathrm{C} 10 \mathrm{C} 9 \\
\mathrm{C} 11 \mathrm{C} 10 \mathrm{C} 9 \mathrm{C} 8\end{array}$} & \multicolumn{2}{|c|}{$\begin{array}{c}\mathrm{C} 10 \mathrm{C} 9 \mathrm{C} 8 \mathrm{C} 7 \\
\text { C9C8C7C6 }\end{array}$} & \multicolumn{2}{|c|}{$\begin{array}{l}\text { C8C7C6C5 } \\
\text { C7C6C5C1 }\end{array}$} \\
\hline 3 & X-ray & 104.3(4) & $-41.3(5)$ & $-64.5(5)$ & $167.2(3)$ & $-70.3(5)$ & $-83.0(5)$ & $165.5(3)$ & $-100.6(4)$ & $65.0(5)$ & $-91.3(4)$ \\
\hline $4 a$ & 22.04 & 108.4 & -36.8 & -63.9 & 166.6 & -73.1 & -82.6 & 167.5 & -97.6 & 58.8 & -90.6 \\
\hline $4 b$ & 22.58 & 57.4 & 55.1 & -143.8 & 68.1 & 66.0 & -179.9 & 63.7 & 78.2 & 62.0 & -55.4 \\
\hline $4 c$ & 23.28 & 97.4 & -44.0 & -68.1 & 167.6 & -89.5 & 89.5 & -167.7 & 68.1 & 44.0 & -97.3 \\
\hline 4d & 23.45 & 53.5 & 68.6 & -64.3 & -61.1 & 162.7 & -162.6 & 61.1 & 64.2 & -68.7 & -53.4 \\
\hline
\end{tabular}

Figure 3. Minimized geometries of [9]3,5-pyridinophane.

The calculations indicate a high conformational mobility of the chain, which is also reflected by the thermal ellipsoids of the crystal structure. The same great mobility was encountered previously in the crystal structure of $(E)-(-)$-2-cyclododecenyl camphanate, which is also loosely packed. ${ }^{4}$ However, the torsion angles are quite different in this case due to the trans double bond, which is a different structural element as compared to our meta-cyclophanic unit.

\section{Experimental Section}

The pyrylium salt with a nonamethylene bridge ${ }^{3}$ was reacted with an excess of an ethanol solution of methylamine to give in $71 \%$ isolated yield the colorless microcrystals of pyridinium salt 3 with a melting point of $229{ }^{\circ} \mathrm{C}$ (dec.). ${ }^{1}$ Crystals suitable for the X-ray analysis were grown by slowly diffusing diethyl ether into a saturated chloroform solution of 3 . The crystal was mounted on a Nonius diffractometer. Details of the crystal structure determination and solution refinement: ${ }^{5-8}$ colourless prism with dimensions $0.525 \times 0.5 \times 0.275 \mathrm{~mm}, M=345.87$, orthorhombic, $P 2{ }_{1} 2{ }_{1}{ }_{1}, a=9.166(1), b=12.759(3), c=15.260(2) \AA, \alpha=\beta=\gamma=90^{\circ}, \mathrm{V}=$ 1784.6(5) $\AA^{3}, Z=4, T=296(2) \mathrm{K}, \rho_{\text {calc }}=1.287, F(000)=744, \mu=0.230 \mathrm{~cm}^{-1}, \lambda=0.71093 \AA$, source $=\operatorname{MoK} \alpha, 2495$ total independent reflections measured, 2305 reflections observed with $I \geq$ $2 \sigma(\mathrm{I})$, no. of parameters refined $=226, R=0.0501$, goodness of fit $=1.129$.

\section{Conclusions}


It is interesting to note that crystal packing effects do not enforce a conformation of the nonamethylene bridge different from the one that lies lowest in energy according to our $\mathrm{MM}+$ calculations, though other conformations are within less than $1.5 \mathrm{kcal} / \mathrm{mol}$, well within the span of such crystal lattice effects. Furthermore, in accord with the earlier published results from DNMR spectra in $\mathrm{CD}_{2} \mathrm{Cl}_{2}$ solution where quite tight ion pairing should be encountered, there were no significant differences in the barrier height encountered for the neutral pyridine 2 and the salts 1 and 3. Therefore, the dynamic processes involve flipping of the meta-cyclophanic ring relative to the ansa-bridge (or vice versa) without involvement the perchlorate counterions. „Hula hoop“ or „cord skipping“ motions do not appear to be involved, since these dynamic processes are likely to require higher barriers in the ionic compounds than would be encountered in 2 .

\section{Acknowledgements}

The measurement was performed in FG Strukturforschung, FB Materialwissenschaft, Technische Hochschule Darmstadt, Petersenstr. 20, 64287 Darmstadt. TSB thanks the Alexander von Humboldt Foundation for a research fellowship at the Technische Hochschule Darmstadt.

\section{References}

1. Balaban, A. T.; Balaban, T. S.; Nitta, M.; Kanomata, N. Bull. Soc. Chim. Belg. 1992, 101, 1047.

2. Hypercube Inc. HyperChem ${ }^{\circledR} 1999$, Release 6.

3. Balaban, A. T. Tetrahedron Lett. 1986, 4643.

4. Ito, S.; Ziffer, H.; Silverton, J. V. Acta Cryst. 1989, C 45, 515.

5. Nonius diffractometer control software, release 5.1. 1993, Nonius GmbH, Solingen, Germany.

6. Sheldrick, G.M. 1993. SHELXL93. Program for crystal structure determination, Univ. of Göttingen, Germany

7. Sheldrick, G.M. 1986. SHELXS86. Program for the solution of crystal structures, Univ. of Göttingen, Germany.

8. Stoe-REDU4, Data Reduction Program, version 6.2c 1987, Stoe \& Cie GmbH, Darmstadt, Germany. 